NIHON REOROJĪ GAKKAISHI Vol. 24, No. 2, 63 66 (1996)

(C)1996 The Society of Rheology, Japan

\title{
論文
}

\section{Drying and Compression under Confining Pressure Effects \\ on Structural Rearrangement of Wood}

\author{
A. Oudjehane ${ }^{\dagger}$ and M. Fushitani ${ }^{*}$
}

†Université de Bourgogne, Département I. E. M., Dijon, France,

*Tokyo University of Agriculture and Technology, Tokyo, Japan

(Received: Jan 20, 1995)

On account of their oriented and heterogeneous structure woods are anisotropic but also porous and thus sensitive to any variation of humidity. Under permanent solicitations (deformations or heat and mass transfer), woods generally show a high degree of directionality and their internal structures evolve and sometimes change. We have observed the evolution of the anisotropy of the structure of oakwood regarding to its response under thermomechanical solicitations: a drying set and triaxial tests.

Key Words: Anisotropy/Oriented tests/Noncrystalline solids

\section{INTRODUCTION}

The complexity of wood structure is recognized from its ultimate particle (cells) to the organization and the compounds of the cells that constitute the trunk which is the solid matter of a tree. Regarding the growth of a tree in length and diameter, the arrangement of the structure at a macroscopical level of a tree trunk confers to wood an initial anisotropy that is transversely isotropic around the trunk axis. However, to this initial anisotropy of structure can be superposed heterogeneities resulting from local growth anomalies like; eccentricity of the heart of the tree, undulation of the annual rings, fractures, knots, and others.

It is useful, for a detailed description of the structure of wood, to make the observations along privileged sections (transversal, radial and tangential) according to three directions; longitudinal along the trunk axis, radial and tangential to the annual rings(Fig. 1). The choice of three privileged sections foreshadows the existence of three orthogonal planes of symmetry. We, therefore, consider oakwood as orthotropic according to the symmetries within the group theory.

†present Address: Department of Wood Seience, Faculty of Forestry, University of British Columbia, 2357 Main Mall, Vancouver, BC, V6T 124 CANADA
At a microscopical level, the cellular organization of deciduous trees such as oak reveals three kinds of cells which arrange along the trunk axis (fibers and vessels)

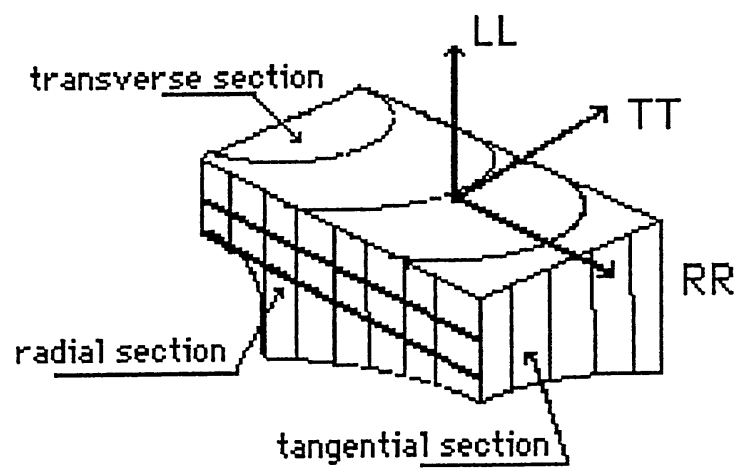

Fig. 1 Privileged sections and directions.

and along the perpendicular direction (vascular rays) confers to oakwood an initial anisotropy within three perpendicular directions that define three orthogonal planes. So, even locally at a microscopical level, oakwood's initial anisotropy of structure is orthotropic.

Wood is also a porous and hygroscopic media thus sensitive to any variation of the moisture content. The influence of the fluid phase (water) upon the behavior of a porous media can be attributed to an interstitial or swell- 


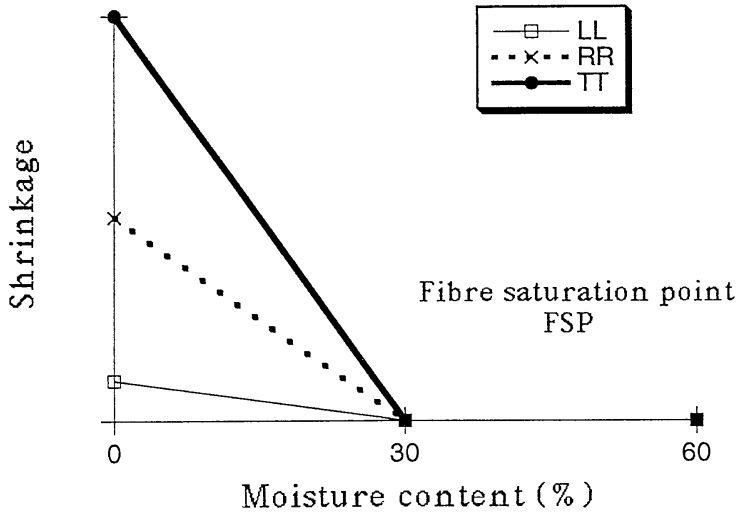

Fig. 2 Anisotropy of shrinkage.

ing pressure. Therefore, absorption induces swelling while a drying leads to shrinkage.

Water contained within woods exists in two states: "free water" retained by capillary forces and "tied water" retained by intermolecular forces.

During the drying of woods, it was measured and observed that below $30 \%$ of moisture content only tied water subsits and shrinkage strain appears ${ }^{1)}$. As shown in Fig. 2,

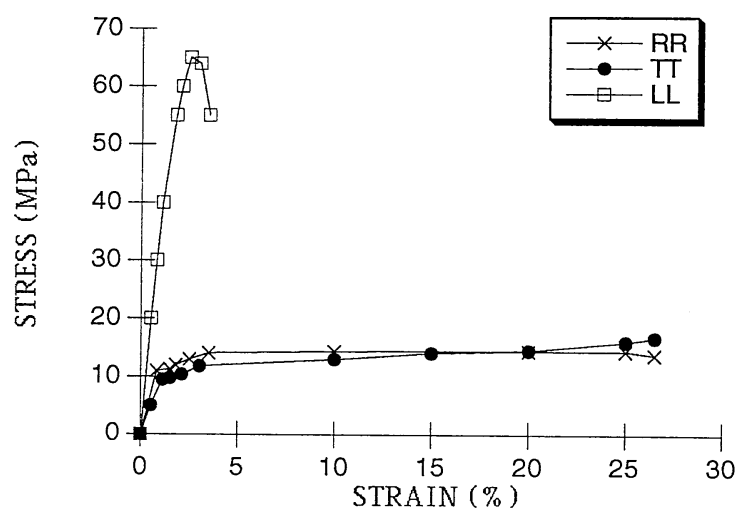

Fig. 3 Mechanical anisotropy.

according to Guitard et al. ${ }^{2)}$, the relation between the shrinkage strain and the variation of moisture content is linear ${ }^{3)}$.

The representation of the shrinkage within the privileged directions previously defined as longitudinal, radial and tangential directions displays the induced shrinkage anisotropy of oakwood.

The mechanical anisotropy of oakwood as shown in Fig. 3 is displayed by the stress-strain curve of compression tests of non-dried oakwood samples at an average moisture content of $30 \%{ }^{4}$.

The compression tests were realized within the three privileged directions of the structure: Radial (RR), tangential (TT) and longitudinal (LL). Hence, the hypothesis of transverse isotropy can not be applied to oakwood's structure according to the fact that the plane RT is not isotropic since we do not observe the same behavior in the radial and tangential directions RR and TT.

The experimental behavior of oakwood is presented through the influence of drying and triaxial tests. The two processes are first detailed in the description of the experimental settings. The effects of both the drying process and triaxial tests are then compared for the conclusion.

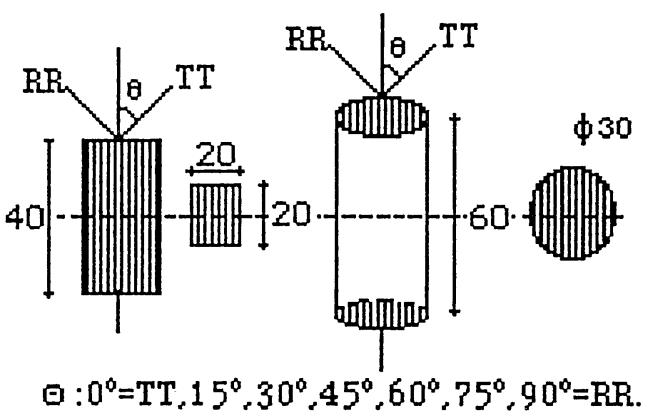

Fig. 4 Shape and dimension of samples.

\section{EXPERIMENTAL SETTINGS}

For the experimental settings, oakwood samples of a high quality oakwood of the forest of Tronçais in France, in the shape of parallelepipeds but also with a cylindric regular shape, were chopped within seven directions regarding the privileged orthogonal directions $\left(\mathrm{TT}=0^{\circ}, \mathrm{RR}\right.$ $=90^{\circ}$ ) respectively the tangential and radial directions to the annual rings. The restriction of the experimental observation to the transverse privileged plane RT, was due to the insensitiveness of oakwood to any variation of moisture content along the fibre within the longitudinal direction. As illustrated in Fig. 2, the shrinkage occurs principally in the radial and tangential directions.

\section{Drying process}

Samples in the shape of parallelepipeds $(20 \times 40 \mathrm{~mm})$ within 7 directions in the plane RT, at the moisture content of $30 \%$, were either directly tested in uniaxial compression or first dried at $102^{\circ} \mathrm{C}$ in an oven, to bring down the moisture content to 20 and $12 \%$, before being tested in uniaxial compression. Four to five tests were performed for each of the 7 directions at three moisture contents.

\section{Triaxial tests}

Samples with a cylindric regular shape $(60 \times \phi 30 \mathrm{~mm})$ also within 7 directions in the plane RT, at the moisture content of $30 \%$, were directly tested in compression under confining pressure without drying but with respect to three lateral pressures $(P=0,10$ and $20 \mathrm{MPa})$. As for the influence of drying, four to five tests were performed for 


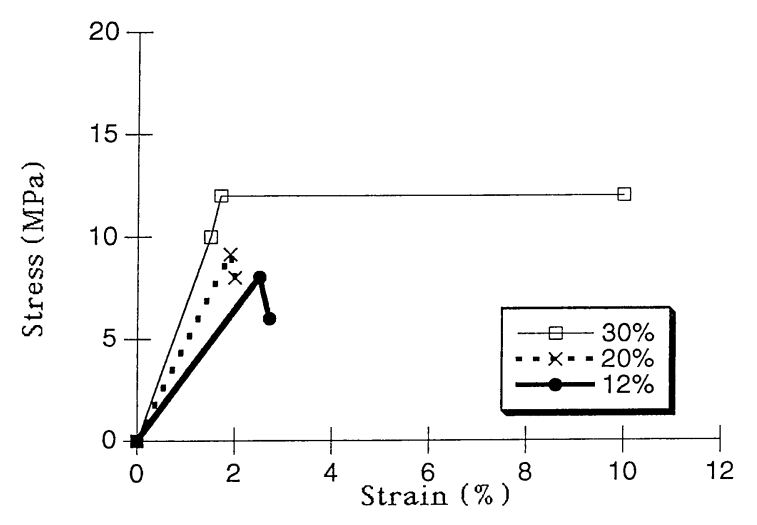

Fig. 5 Influence of drying (Radial direction $90^{\circ}$ )

each of the 7 directions at three lateral pressures and we analysed the rheological behavior and the mechanical anisotropy according to the stress-strain curve and the evolution of the elastic moduli ratio curves respectively, ragarding the three lateral pressures.

\section{EXPERIMENTAL RESULTS}

\section{Drying process}

Through the analysis of the stress-strain curve, we got the rheological behavior of dried and non-dried oakwood. The mechanical anisotropy of deformation is displayed by

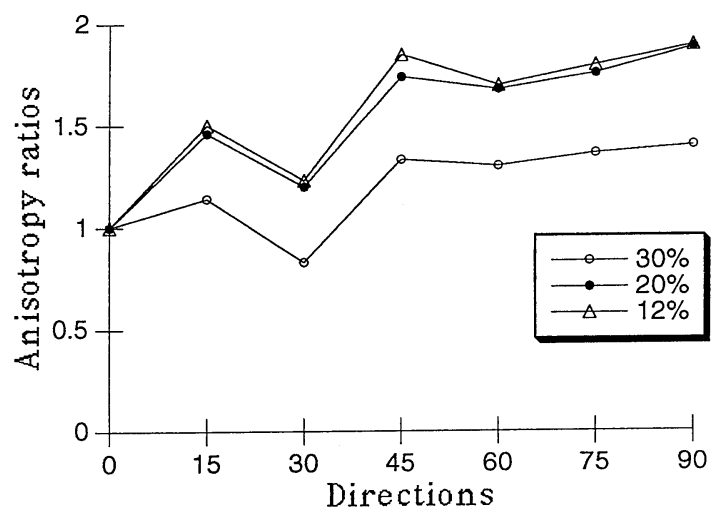

Fig. 6 Elastic moduli anisotropy. Plane (RT). Drying process.

the evolution of the elastic moduli ratio according to both the orientation of the samples in the plane RT and the moisture content.

The stress-strain curves of the rheological behavior of oakwood, resumed in Fig. 5, revealed that non-dried oakwood $(30 \%)$ is elastoplastic and perfectly plastic beyond the yield stress while dried oakwood (20\% and $12 \%$ ) presents a brittle fracture preceeded by linear elastic deformation.

As we performed it, the drying process induces a decrease of the samples elastic moduli. Dried samples are thus, less elastic than non-dried ones.

The induced anisotropy of the elastic moduli due to the drying process is represented by the evolution of the elastic moduli ratio $E / E_{0}$ according to the orientation of the samples in the plane RT at three moisture contents. $E_{0}$ and $E$ are the elastic moduli in the direction $0^{\circ}$ (TT) and any other direction within the plane $\mathrm{RT}$ ( $\mathrm{RR}$ at $90^{\circ}$ ) respectively.

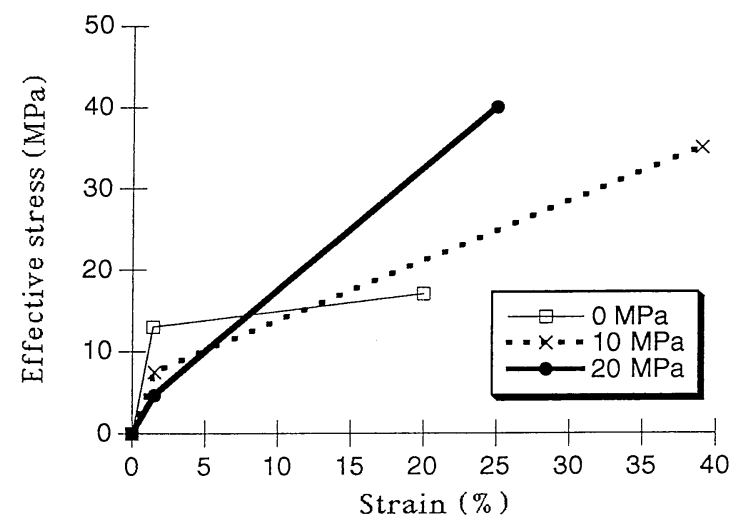

Fig. 7 Influence of the confining pressure (Radial direction $90^{\circ}$ ).

First, we remark that the mechanical anisotropy of oakwood is discontinuous with singularities at particular directions around $30^{\circ}, 45^{\circ}$ and $60^{\circ}$. Second, we notice that the induced anisotropy by the drying process, at $20 \%$ and

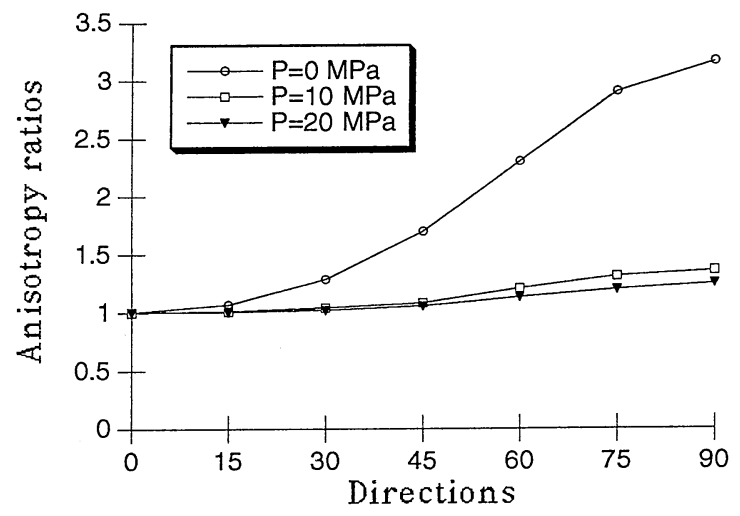

Fig. 8 Elastic moduli anisotropy. Plane (RT). Triaxial tests.

$12 \%$ of moisture contents, conserves the type of the initial anisotropy at $30 \%$ of humidity. But, though the type of anisotropy is conserved, the anisotropy ratios of the elastic moduli increase when wood is dried while the elastic moduli decrease with the drying $\left(E_{90} / E_{0}=2\right.$ at $20 \%$ and $12 \%$, while $E_{90} / E_{0}=1.4$ at $30 \%$ ).

Triaxial tests

A short outline of the rheological behavior of oakwood 
regarding compression tests under confining pressures is illustrated in the stress-strain curve (Fig. 7 ). Oakwood is essentially elastic and perfectly plastic in the privileged radial and tangential directions. For all that, the behavior is more generally elastic and perfectly plastic for all "offaxis" orientations at the pressure $P=0 \mathrm{MPa}$. The plastic flow evolves with the lateral pressure and thus the behavior of oakwood tends to be elastic with strain hardening at the pressures $P=10$ and $20 \mathrm{MPa}$.

The influence of the confining pressure upon the mechanical anisotropy of oakwood is shown by the evolution of the elastic moduli ratios $E / E_{0}$, according to the orientation of the samples in the plane RT and to the three lateral pressures.

Regarding Fig. 8, the elastic anisotropy of oakwood and its mechanical behavior are strongly influenced under the confining pressure. The initial elastic anisotropy in the plane (RT) at $P=0 \mathrm{MPa}$, tends to be almost isotropic at $P$ $=20 \mathrm{MPa}$, thus the evolution of both the type and the degree of the elastic anisotropy of oakwood is observed. This result can be relied on the irreversible deformations that we observed on the samples tested at the confining pressures $P=10$ and $20 \mathrm{MPa}$ : the samples pulled out from the triaxial cell, initially straight circular cylinder, showed inclinations and elliptic transversal sections.

Since principal directions of the strain tensor and symmetry axes of the samples do not coincide, the irreversible deformations are due to a distortion strain. Therefore inclinations of samples can be associated to the orientation of the symmetry axes of the samples regarding the privileged directions of oakwood in the plane RT $\left(0^{\circ} \mathrm{TT}\right.$ or $\left.90^{\circ} \mathrm{RR}\right)$. Hence, a structural rearrangement with different material symmetry is displayed through evolution of anisotropy.

\section{DISCUSSION}

Elastic and perfectly plastic, elastic and brittle or elastoplastic with strain hardening are the rheological models of oakwood depending on whether it is non-dried, dried or tested at higher pressures in triaxial tests. With regard to its constitution, the internally oriented structure of oakwood is woody as a stratified and expended fibrous composite: crystalline fibers and polymeric matrix created in equal parts by amorphous (lignin) and semicrystalline (hemicellulose) polymers.

The response of polymers to an applied stress depends on their degree of crystallinity. Crystalline polymers are generally stiffer and more resistant to permanent deformation than non-crystalline ones. Amorphous-crystalline polymers mixtures display a higher strength as the crystallinity increases and conversely, though permanent deformations of both crystalline and non-crystalline polymers are related to the ease of interchain sliding that induces a structural rearrangement. It is thus, possible to conclude that in a general state of stress such as triaxial tests, the structure arrangements of oakwood evolve through a more ordered arrangement of molecular chains. This was reflected at a macroscopic level by irreversible deformation of oakwood's specimens (inclinations and elliptic cross-sections) and by the induced anisotropy of the elastic moduli ratios according to the confining pressure. The induced and developed new mechanical anisotropy can thus be likened to isotropy of oakwood in the plane RT (cf Fig. 8), with new material symmetry.

Drying process inducing conserved initial anisotropy type but brittle behavior (cf Fig. 5) is indeed due to damage of oakwood. Mechanical damage can be attributed to crazing mechanism initiated with the elastic deformations and occuring, at a stress level below the normal strength of non-dried samples, with a sample brittle fracture. Such deformations displayed generally in non-crystalline polymers, it is indeed that in the structure of dried oakwood, the crystalline-amorphous region have less crystalline amount set although more amorphous and thus rather induces less resistance and permanent deformations.

Although phenomenologic since made at a macroscopic level, the results stated above outlined how complex the structure of oakwood is. Our works displayed how distinguished non-linear deformations, damage and strain hardening can be induced by oriented rearrangement of the internal structure.

\section{REFERENCES}

1) Kollman FP, Cote WA, "Principles of wood science and technology”, Vol 1, Springer Verlag ed (1968).

2) Guitard D, Genevaux JM, Arch Mechanics, 40, 5-6, 665 (1988).

3) Sales C, "Contribution a l'analyse des contraintes de séchage dans le bois", Thesis INPL NANCY (1988).

4) Oudjehane A, "Contributions theóriques et expérimentales à l'étude du comportement de milieux poreux anisotropes: le bois", Thesis Université Blaise Pascal Clermont II (1992).

5) Courtney $\mathrm{TH}$, "Mechanical behavior of materials", McGraw-Hill ed (1990), Chap 8, p325.

6) Lemaitre J, Chaboche JL, "Mecanique des matériaux solides”, Dunod ed (1985).

7) François D, Pineau A, Zaoui A, "Comportement mécanique des matériaux”, Vol 1, Hermes ed (1992).

8) Doi N, Fushitani M, Kaburagi J, J wood res soc, Japan, vol 24, 4, 217 (1978).

9) Eringen C, "Continuum Physics", Vol II, Academic Press ed (1975). 